\title{
SENSITIVE GANGLIONEURONOPATHY WITHOUT SICCA SYMPTOMS AS INITIAL PRESENTATION OF SJOGREN SYNDROME: A CASE REPORT
}

Lorena Elizabeth Betancourt (HC-FMUSP, São Paulo, SP, Brasil), Erivelton Azevedo Lopes (HC-FMUSP, São Paulo, SP, Brasil), Valdirene Silva Siqueira (HC-FMUSP, São Paulo, SP, Brasil), Felipe Freire Silva (HCFMUSP, São Paulo, SP, Brasil), Bruna Giusto Bunjes (HC-FMUSP, São Paulo, SP, Brasil), Douglas Amaral

Moreira (HC-FMUSP, São Paulo, SP, Brasil), Ari Stiel Radu Halpern (HC-FMUSP, São Paulo, SP, Brasil), Lissiane Karine Noronha Guedes (HC-FMUSP, São Paulo, SP, Brasil), Sandra Gofinet Pasoto (HC-FMUSP, São Paulo, SP, Brasil)

\section{BACKGROUND}

Acquired sensitive ganglioneuronopathy $(\mathrm{GN})$ is a rare subgroup of periphery neuropathy characterized by lesion of the neuronal body of the dorsal root. It's generally associated to conditions like infections, neoplasms and autoimmune diseases. (GN) is a recognized complication of Sjogren's syndrome (SS). The glandular abnormalities are minimal or absent in patients with $\mathrm{GN}$ and the diagnosis of the underlying condition is often missed. Here, we reported a case of GN without sicca symptoms from our tertiary hospital.

\section{CASE REPORT}

A caucasian 30-years-old healthy female with history of distal progressive and asymmetrical paresthesias in limbs for 3 years, developed over the four-year hypoesthesia in both hands with difficulty for writing and typing, besides that progressive and incapacity imbalance; through the time of these symptoms; dry mouth, dry eye, mumps and other alterations was absent. The neurological examination showed a diffuse hyporreflexia, reduction of deep sensitivity, ataxia with muscular strength preserved, receiving the hypothesis of SN. Infectious, metabolic, hormonal and neoplastic causes were excluded. Laboratory tests showed anemia with normal acute phase reactants. Arterial blood gas analysis revealed metabolic acidosis with high urinary $\mathrm{pH}$, characterizing renal tubular acidosis. An antinuclear antibody test exhibited a nucleolar pattern with high title (1:320) with specific SS - Ro positive. In spite of denying symptoms of sicca syndrome, ocular and saliva tests were performed without any alterations. Due to the strong clinical suspicion, a salivary gland biopsy was performed, showing a lymphocytic sialoadenitis with a focal score of 3.8, compatible with SS. We introduced high doses of methylprednisolone and intravenous cyclophosphamide for 6 months. After that, rituximab in association with mycophenolate mofetil was started, observing complete remission of neurological symptoms and she was able to return to their usual activities.

\section{CONCLUSION}

Ganglioneuronopathy (GN) is a rare but recognized involvement of Sjogren's syndrome (SS). Because of that it's important to think in SS diagnosis even without sicca syndrome. Considering this, the criteria ACREULAR/2016 is applicable to any patient who had suspicion of SS due to systemic features from ESSDAI measure with at least one positive domain item, even without sicca symptons. This way we can improve a very early diagnosis, institute a promptly treatment and get a better prognosis. 